\section{Novel Anaplasmataceae agents Candidatus Ehrlichia hydrochoerus and Anaplasma spp. Infecting Capybaras, Brazil}

\author{
Thállitha S.W.J. Vieira, Flávia C.M. Collere, \\ Larissa D.R. Ferrari, Rafael A. Baggio, \\ Rogério R. Lange, Marcos V. Ferrari, \\ Juan C.M. Duque, Gustavo S. Sanches, \\ Nathália A. Pereira, Daniel M. Aguiar, \\ Marcelo B. Labruna, Rafael F.C. Vieira
}

Author affiliations: Universidade Federal do Paraná, Curitiba, Brazil (T.S.W.J. Vieira, F.C.M. Collere, L.D.R. Ferrari, R.R. Lange, M.V. Ferrari, J.C.M. Duque, R.F.C. Vieira); Universidade Federal de Minas Gerais, Belo Horizonte, Brazil (R.A. Baggio); Pontifícia Universidade Católica do Paraná, Curitiba (G.S. Sanches); Universidade Federal do Mato Grosso, Cuiabá, Brazil (N.A. Pereira, D.M. Aguiar); Universidade de São Paulo, São Paulo, Brazil (M.B. Labruna); Global One Health initiative, The Ohio State University, Columbus, Ohio, USA (R.F.C. Vieira)

\section{DOI: https://doi.org/10.3201/eid2802.210705}

We amplified Ehrlichia and Anaplasma DNA from Amblyomma dubitatum tick-infested capybaras (Hydrochoerus hydrochaeris) in southern Brazil. Sequencing of 16S rRNA, sodB, and groEL indicated a novel Ehrlichia species, and sequencing of $16 \mathrm{~S}$ rRNA from 2 capybaras indicated a novel Anaplasma species. The tick vectors remain unknown.

E hrlichia and Anaplasma species are tickborne bacteria that infect animals and humans worldwide. To date, 6 Ehrlichia species have been described (E. canis, E. chaffeensis, E. ewingii, E. muris, E. ruminantium, and $E$. minasensis), and 8 Anaplasma species have been described (A. bovis, A. capra, A. centrale, A. marginale, A. odocoilei, A. ovis, A. platys, and A. phagocytophilum). In addition, other native Ehrlichia species have been described in wild animals from Brazil (1).

Although capybaras (Hydrochoerus hydrochaeris), the largest living rodents in the world, have been implicated as a major amplifying host of Rickettsia rickettsii (the etiologic agent of Brazilian spotted fever) for Amblyomma sculptum ticks, studies focusing on other tickborne diseases agents are lacking in this rodent. Accordingly, we conducted a comprehensive survey for the detection of Ehrlichia and Anaplasma species in a population of capybaras from Pinhais Municipality, Paraná State, southern Brazil.
We retrieved blood samples from 17 capybaras and salivary glands from 11 Amblyomma dubitatum ticks from these capybaras that were collected for a previous study conducted in southern Brazil (2). We screened blood samples by using PCR targeting of the 16S rRNA gene of Ehrlichia and Anaplasma $(3,4)$. We then tested samples positive by PCR by using PCR that targeted a fragment of the $d s b$ and $\operatorname{sodB}$ genes of Ehrlichia species $(1,5)$ and the groEL gene of Ehrlichia and Anaplasma species (6). We used blood samples from dogs positive for E. canis as positive controls and nuclease-free water samples as negative controls.

The Ehrlichia 16S rRNA PCR assay yielded amplicons in 16/17 (94.12\% [95\% CI 73.02\%-98.95\%]) capybaras, from which we generated amplicons by the sodB PCR (300 bp) and groEL PCR (1,100 bp) assays. No sample yielded amplicon by the $d s b$ PCR assay. We sequenced amplicons obtained from 4 16S rRNA, 5 sodB, and 4 groEL PCR-positive samples in both directions by using the Sanger method. We submitted all nucleotide sequences obtained to GenBank (Appendix, https://wwwnc.cdc.gov/EID/ article/28/2/21-0705-App1.pdf).

We observed infestations by $A$. dubitatum ticks in all capybaras, from which we collected 26 males, 16 females, and 122 nymphs. Among salivary glands from 11 adult ticks, 1 (9.09\%) tested positive for Ehrlichia species by the $16 \mathrm{~S}$ rRNA PCR. However, multiple attempts to sequence the $16 \mathrm{~S}$ rRNA gene detected in tick salivary glands were unsuccessful because of the faint bands.

We observed neither abnormalities nor inclusionlike bodies of Ehrlichia or Anaplasma during the evaluation of Giemsa-stained thin blood smears of the capybaras. We tested Ehrlichia antibodies in capybara serum samples with an indirect immunofluorescent assay using E. canis (São Paulo and Cuiabá strains) as antigens; serum samples were positive if reacting at a dilution $\geq 1: 40$ (7). A total of 6/17 (35.29\% [95\% CI $17.31 \%-58.70 \%]$ ) capybaras showed antibodies against $\geq 1$ of the E. canis antigens. When we used the Cuiabá strain of E. canis as antigen, 4/17 (23.53\% [95\% CI 9.56\%-47.26\%]) capybaras were seropositive, whereas 6/17 (35.29\%) were positive when we used the São Paulo strain. Four capybaras were seropositive for both E. canis strains. Antibody endpoint titers ranged from 40 to 640 for both E. canis antigens.

According to serologic testing, PCR amplification, and DNA sequencing results, $A$. dubitatum tick-infested capybaras in southern Brazil may be infected with a novel Ehrlichia agent and a novel Anaplasma species. Serologic screening showed 
exposure to Ehrlichia species in 35\% of the capybaras. A previous study failed to detect Ehrlichia DNA in spleen tissue of capybaras from southeastern Brazil (8), and we know of no previous study of Anaplasma species that has been performed in this rodent species.

Partial sequences of $16 \mathrm{~S}$ rRNA and 2 proteincoding genes ( $\operatorname{codB}$ and groEL) obtained from capybaras indicate a novel Ehrlichia species. Partial 16S rRNA gene sequences from capybara no. II showed that the detected Ehrlichia agent shared 95.67\% identity with $A$. phagocytophilum, whereas sequences from capybara no. III showed that the detected Ehrlichia agent shared $94.28 \%$ identity with E. chaffeensis. Partial sodB genes showed $82.23 \%-$ $85.07 \%$ identity with E. chaffeensis or E. ruminantium, whereas partial groEL genes showed identity with $76.52 \%$ with $A$. phagocytophilum. A previous study stated that different bacterial isolates showing $<97 \%$ similarity in the $16 \mathrm{~S}$ rRNA gene belong to different species (9). In addition, protein-coding genes should be used in addition to the 16S rRNA gene for identification of novel species (10). Our genetic findings support the infection of capybaras in Brazil with a novel Ehrlichia species, herein named Candidatus Ehrlichia hydrochoerus (Figure).

Partial sequences of $16 \mathrm{~S}$ rRNA gene obtained from capybaras VI and VII demonstrated a novel Anaplasma species. Partial 16S rRNA gene sequences showed identity of $96.76 \%$ with Anaplasma sp. detected in dogs from the Philippines and $97.93 \%$ with $A$. phagocytophilum, with $100 \%$ query coverage. Bayesian inference showed that the capybara Anaplasma species detected was related to $A$. odocoilei from North America, which indicates a novel Anaplasma species infecting capybaras in Brazil.

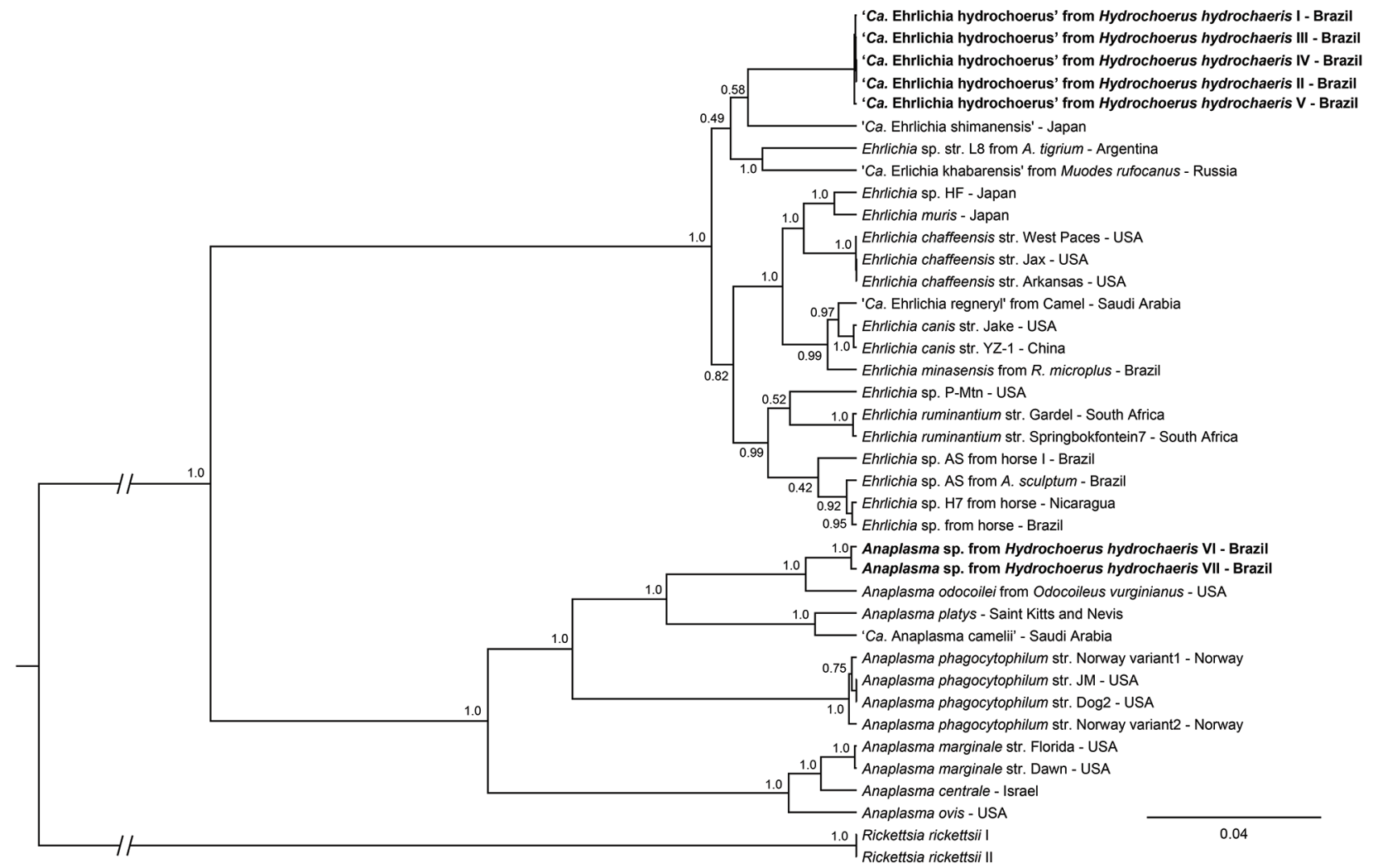

Figure. Phylogenetic analysis of $16 \mathrm{~S}$ rRNA, sodB, and groEL partial sequences of Candidatus Ehrlichia hydrochoerus and Anaplasma spp. obtained from capybaras (Hydrochoerus hydrochaeris), southern Brazil. These sequences (in bold) and those of other Ehrlichia and Anaplasma species were aligned using MAFFT 7.110 (https://mafft.cbrc.jp/alignment/server). Phylogenetic analyses of each gene were based on Bayesian inference using Beast version 1.8.4 (https://beast.community/index.html). We performed 3 independent runs of 100 million generations of Monte Carlo Markov chain with 1 sampling/10,000 generations and a $10 \%$ burn-in. We estimated substitution models as generalized time reversible plus gamma for $16 \mathrm{~S}$ rRNA (A), Hasegawa-Kishino-Yano plus gamma for sodB (B), and Tamura-Nei plus gamma for groEL (C) genes on the basis of Akaike information criterion by using jModeltest version 2.1.10 (https:// github.com/ddarriba/jmodeltest2/releases/tag/v2.1.10r20160303). The tree was rooted with Rickettsia rickettsii (GenBank accession nos. CP000766.3 and CP018913.1). Complete GenBank accession numbers are listed in the Appendix (https://wwwnc.cdc.gov/EID/ article/28/2/21-0705-App1.pdf). Scale bar indicates number of substitutions per site. Ca., Candidatus. 


\section{Acknowledgments}

We thank Hubert D. Fanien for providing O'TOM/

Tick Twister.

The Fundação Araucária (grant no. 09/2016) and the Brazilian National Council of Scientific and Technological Development (grant no. 425597/2018-0) provided financial aid and support to carry out this research. The Brazilian National Council of Scientific and Technological Development also provided research fellowships to D.M.A. (grant no. 303677/2018-0), M.B.L. (grant no. 301641/2019-6), and R.F.C.V. (grant no. 313161/2020-8). F.C.M.C. was sponsored by a fellowship from the Coordenação de Aperfeiçoamento de Pessoal de Nível Superior at the time of this study.

\section{About the Author}

Dr. Vieira is a research associate at the Vector-borne Diseases Laboratory, Universidade do Paraná, Curitiba, Brazil, and specializes in tickborne diseases. Her research is focused on molecular characterization of Anaplasmatacae in Brazil.

\section{References}

1. Almeida AP, Souza TD, Marcili A, Labruna MB. Novel Ehrlichia and Hepatozoon agents infecting the crab-eating fox (Cerdocyon thous) in southeastern Brazil. J Med Entomol. 2013;50:640-6. https://doi.org/10.1603/ME12272

2. Vieira RFC, Santos NJR, Valente JDM, Santos LP, Lange RR, Duque JCM, et al. 'Candidatus Mycoplasma haematohydrochoerus', a novel hemoplasma species in capybaras (Hydrochoerus hydrochaeris) from Brazil. Infect Genet Evol. 2021;93:104988. https://doi.org/10.1016/ j.meegid.2021.104988

3. Parola P, Roux V, Camicas JL, Baradji I, Brouqui P, Raoult D. Detection of Ehrlichiae in African ticks by polymerase chain reaction. Trans R Soc Trop Med Hyg. 2000;94:707-8. https:/ / doi.org/10.1016/S0035-9203(00)90243-8

4. Ruiz-Fons F, Fernández-de-Mera IG, Acevedo P, Gortázar C, de la Fuente J. Factors driving the abundance of Ixodes ricinus ticks and the prevalence of zoonotic I. ricinus-borne pathogens in natural foci. Appl Environ Microbiol. 2012;78:2669-76. https:/ / doi.org/10.1128/AEM.06564-11

5. Qurollo BA, Davenport AC, Sherbert BM, Grindem CB, Birkenheuer AJ, Breitschwerdt EB. Infection with Panola Mountain Ehrlichia sp. in a dog with atypical lymphocytes and clonal T-cell expansion. J Vet Intern Med. 2013;27:1251-5. https://doi.org/10.1111/jvim.12148

6. Barber RM, Li Q, Diniz PP, Porter BF, Breitschwerdt EB, Claiborne MK, et al. Evaluation of brain tissue or cerebrospinal fluid with broadly reactive polymerase chain reaction for Ehrlichia, Anaplasma, spotted fever group Rickettsia, Bartonella, and Borrelia species in canine neurological diseases (109 cases). J Vet Intern Med. 2010;24:372-8. https:// doi.org/10.1111/j.1939-1676. 2009.0466.x

7. Aguiar DM, Cavalcante GT, Pinter A, Gennari SM, Camargo LM, Labruna MB. Prevalence of Ehrlichia canis (Rickettsiales: Anaplasmataceae) in dogs and Rhipicephalus sanguineus (Acari: Ixodidae) ticks from Brazil. J Med Entomol. 2007;44:126-32. https://doi.org/10.1093/ jmedent/41.5.126

8. Labruna MB, McBride JW, Camargo LM, Aguiar DM, Yabsley MJ, Davidson WR, et al. A preliminary investigation of Ehrlichia species in ticks, humans, dogs, and capybaras from Brazil. Vet Parasitol. 2007;143:189-95. https://doi.org/ 10.1016/j.vetpar.2006.08.005

9. Drancourt M, Raoult D. Sequence-based identification of new bacteria: a proposition for creation of an orphan bacterium repository. J Clin Microbiol. 2005;43:4311-5. https:/ / doi.org/ 10.1128/JCM.43.9.4311-4315.2005

10. Fournier PE, Dumler JS, Greub G, Zhang J, Wu Y, Raoult D. Gene sequence-based criteria for identification of new rickettsia isolates and description of Rickettsia heilongjiangensis sp. nov. J Clin Microbiol. 2003;41:5456-65. https:// doi.org/10.1128/JCM.41.12.5456-5465.2003

Address for correspondence: Thállitha S.W.J. Vieira, Departamento de Medicina Veterinária, Campus Agrárias, Universidade Federal do Paraná. R. dos Funcionários, 1540, Juvevê, Curitiba, PR, 80035-050, Brazil; email: thallitha.vieira@ufpr.br

\section{Dirofilaria immitis Pulmonary Dirofilariasis, Slovakia}

\author{
Martina Miterpáková, Daniela Antolová, \\ Jana Rampalová, Miroslava Undesser, \\ Tomáš Krajčovič, Bronislava Víchová
}

\begin{abstract}
Author affiliations: Institute of Parasitology of Slovak Academy of Sciences, Košice, Slovakia (M. Miterpáková, D. Antolová, B. Víchová); Unilabs Slovakia Ltd., Diagnostic Center of Pathology, Bratislava, Slovakia (J. Rampalová, T. Krajčovič); Ambulance of Pneumology and Phthisiology, Dunajská Streda, Slovakia (M. Undesser)
\end{abstract}

DOI: https://doi.org/10.3201/eid2802.211963

Dirofilaria immitis is a parasite related to pulmonary dirofilariasis in humans, its accidental hosts. We detected an autochthonous case of $D$. immitis infection in a woman from Slovakia. The emergence and spread of this parasite in Europe indicates a critical need for proper diagnosis of infection. 